\title{
Shaping the third-harmonic radiation from silicon nanodimers
}

\author{
Lei Wang, ${ }^{a}$ Sergey Kruk, ${ }^{a}$ Lei Xu, ${ }^{\text {a,b }}$ Mohsen Rahmani, ${ }^{a}$ Daria Smirnova, ${ }^{a, c}$ \\ Alexander Solntsev, ${ }^{a}$ Ivan Kravchenko, ${ }^{d}$ Dragomir Neshev ${ }^{a}$ and Yuri Kivshar*a
}

Recent progress in the study of resonant light confinement in high-index dielectric nanostructures suggests a new route for achieving efficient control of both electric and magnetic components of light. It also leads to the enhancement of nonlinear effects near electric and magnetic Mie resonances with an engineered radiation directionality. Here we study the third-harmonic generation from dimers composed of pairs of two identical silicon nanoparticles and demonstrate, both numerically and experimentally, that the multipolar harmonic modes generated by the dimers near the Mie resonances allow the shaping of the directionality of nonlinear radiation.

Nonlinear nanophotonics has attracted a lot of attention due to the fact that a small volume of nanoparticles can create surprisingly strong nonlinear effects. ${ }^{1-3}$ This is at the heart of modern nanophotonics that aims toward efficient light manipulation at the nanoscale and a design of ultrafast compact optical devices for fully-functional photonic circuitry and advanced metadevices. ${ }^{4,5}$

For many years, nanoplasmonics has been a driving force to achieve the enhancement of nonlinear effects in nanoscale structures. ${ }^{1,2}$ In particular, the observation of the third-harmonic generation (THG) was reported for gold colloids, ${ }^{6}$ metallic nanoantennas, ${ }^{7}$ and complex plasmonic structures. ${ }^{8}$ It was also shown that the third-harmonic signal can be enhanced in nanoparticle dimers composed of a pair of identical metallic nanoparticles due to the field enhancement in a gap separating the nanoparticles. ${ }^{9-11}$ However, metallic structures have limitations in their efficiency and functionalities at optical frequencies imposed by Ohmic losses, small mode volumes and low laser damage threshold. This limits the further progress and applications of nonlinear optics at the nanoscale.

Dielectric resonant nanostructures can overcome many of such problems since their optical losses at visible and near-IR

\footnotetext{
${ }^{a}$ Nonlinear Physics Center, Research School of Physics and Engineering, Australian National University, Canberra ACT 2601, Australia.

E-mail: yuri.kivshar@anu.edu.au

${ }^{b}$ The MOE Key Laboratory of Weak Light Nonlinear Photonics, School of Physics and TEDA Applied Physics Institute, Nankai University, Tianjin 300457, China

${ }^{c}$ Institute of Applied Physics, Nizhny Novgorod 603950, Russia

${ }^{d}$ Center for Nanophase Materials Sciences, Oak Ridge National Laboratory, oak Ridge, TN 37831, USA
}

wavelengths are almost negligible. ${ }^{12}$ Such nanoparticles can support electric and magnetic Mie-type resonances enabling directional scattering of light by compact optical nanoantennas. ${ }^{13-17}$ For dielectric nanoparticles, nonlinear effects can be enhanced substantially near the resonances which may involve a higher-order multipolar response. ${ }^{18-22}$ The interference of these multipoles defines the radiation pattern efficiency of the nonlinear process. $^{3}$

Silicon (Si) nanoparticles were first explored for nonlinear effects at the nanoscale due to their large third-order nonlinear susceptibilities. ${ }^{18,23,24}$ The study of THG from isolated silicon nanodisks revealed that the field localization at the magnetic resonance can result in two orders of magnitude enhancement of the harmonic intensity with respect to unstructured bulk silicon. ${ }^{18,24}$ Later, third-harmonic was studied in Ge nanodiscs. ${ }^{25}$ The study revealed the importance of interference effects. In particular, it demonstrated the enhancement of the third harmonic by the excitation of the near-field anapole mode. These findings suggest novel opportunities for engineering the nonlinear response at the nanoscale with dielectric resonant nanostructures.

Pairing up two isolated nanoparticles and creating dimer nanoantennas enrich multipolar behavior in both linear and nonlinear regimes. The effects of multipolar mode decomposition on linear optical properties have recently been reported by several groups. ${ }^{16,17,26-32}$ It was observed that coupling between the resonant nanoparticles placed in a close proximity allows for a substantial enhancement of both electric and magnetic fields via hybridization of individual electric and magnetic modes, and it gives an additional degree of freedom to engineer the directional scattering. However, the effect of hybridized modes supported by dielectric dimers on their nonlinear optical properties remains unknown. 


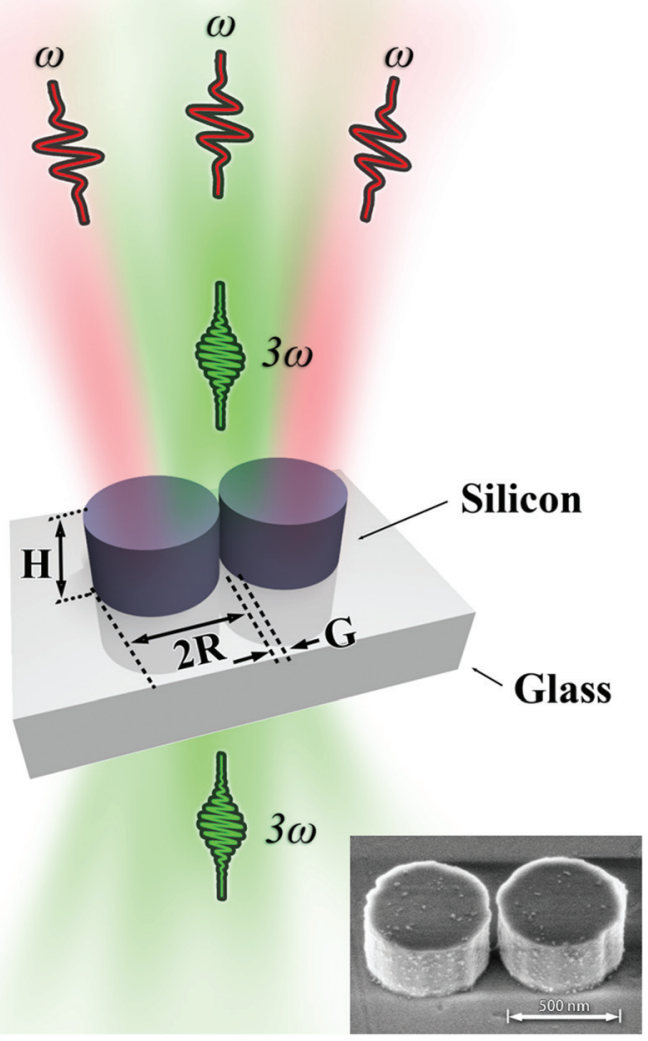

Fig. 1 Schematic of a third-harmonic generation in our system. A dimer nanoantenna is composed of two silicon nanodisks. Insert: SEM image of the dimer.

Here we provide numerical and experimental studies of THG from symmetric silicon nanodimers. We demonstrate the control of the directionality of the third-harmonic radiation due to the multipolar interference effects and near-field enhancement. More specifically, we demonstrate that geometry-dependent multipolar modes in silicon dimers allow one to effectively shape and switch the nonlinear third harmonic radiation pattern, its intensity and directionality, as presented schematically in Fig. 1 where an insert shows an SEM image of one of the fabricated Si dimer samples.

\section{Experimental and numerical techniques}

We fabricate the dimer samples from polycrystalline silicon on a fused silica substrate $(500 \mu \mathrm{m})$ using electron-beam lithography (EBL). Each nanodimer is created by two identical silicon nanodisks, as shown in the insert in Fig. 1. We fabricate a wide range of dimers with varying disk radii ranging from $200 \mathrm{~nm}$ to $300 \mathrm{~nm}$. The dimers have an identical height of $H=$ $300 \mathrm{~nm}$, and each dimer has a well-controlled gap between the neighboring of $G=20 \mathrm{~nm}$. For the nonlinear experiments, we place an isolated nanodimer in a focal spot of two confocal air objective lenses. The numerical aperture of these lenses corresponds to the collection angles for the third-harmonic emission within approximately 58 and 64 degrees in air, respectively. We optically pump the dimer with a femtosecond laser with $1556 \mathrm{~nm}$ central wavelength and control the pump polarization. We use two cooled CCD cameras to detect the thirdharmonic radiation, its power and directionality diagram. More details of this method of optical diagnostics can be found in ref. 22.

To design dimers and predict their linear and nonlinear optical properties, we apply the wavelength-dependent complex-value refractive index of silicon, ${ }^{33}$ and perform numerical simulations using the finite element method (FEM) solver of COMSOL Multiphysics, following the method described in ref. 22-24. To achieve high nonlinear THG conversion with a single symmetric silicon dimer, we are primarily guided by the resonant enhancement of the local fields at the fundamental wavelength (FW). Therefore, we first study the linear scattering characteristics at our laser operating wavelength $1556 \mathrm{~nm}$. To optimize the geometry, we scan the radius $R$ for a fixed disk thickness of $H=300 \mathrm{~nm}$ and a constant interparticle gap $G=20 \mathrm{~nm}$, as shown in Fig. 2. The plotted scattering efficiency per disk is defined as $Q_{\mathrm{sca}}=C_{\mathrm{sca}} / S_{0}$, where $C_{\mathrm{sca}}$ is
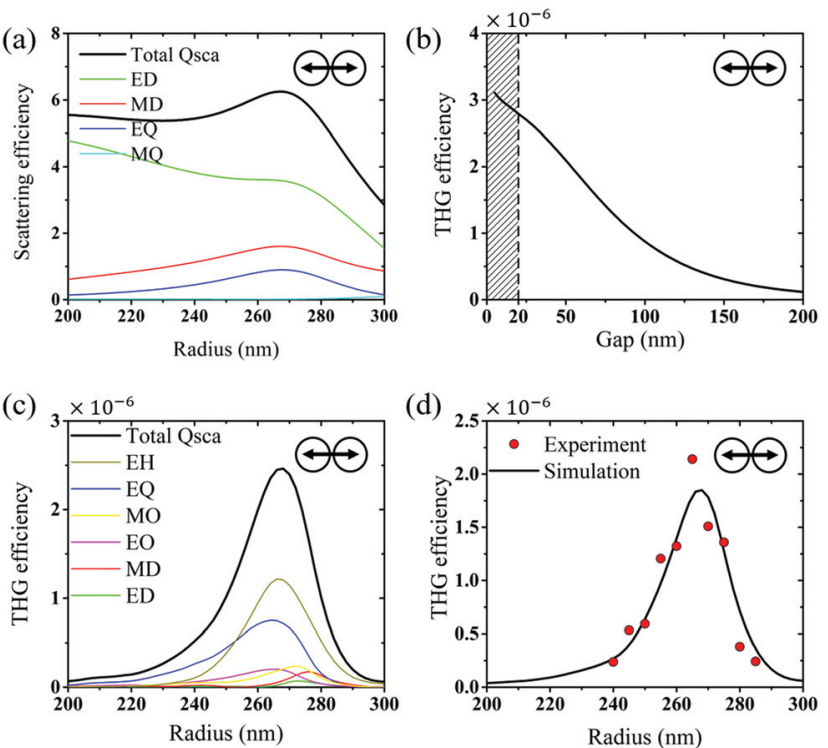

Fig. 2 (a) Calculated total linear scattering efficiency (black curve) of the silicon dimer nanoantenna with fixed gap size $G=20 \mathrm{~nm}$ and varying disk radius. Contributions of leading multipoles in the multipolar decomposition of the calculated electromagnetic field are shown in colored curves. (b) Dependence of the THG conversion efficiency on the gap size at fixed radius $R=270 \mathrm{~nm}$. (c) Calculated THG efficiency versus radius and its multipole contributions. (d) Experimentally measured (dots) and calculated (line) portion of the total THG efficiency captured by the two collecting objectives. The results shown are for the fixed gap $G=20 \mathrm{~nm}$ and varying disk radius. Abbreviations: $\mathrm{E}$ - electric, $\mathrm{M}$ - magnetic, D - dipole, Q - quadrupole, O - octupole, H - hexadecapole. Insets show the polarization of the pump. 
the scattering cross section and $S_{0}=\pi R^{2}$ is the area of the disk. We further characterize the optical scattering features by multipolar expansion.

We perform the multipolar decomposition using the polarization currents induced inside the nanoparticles. We choose this method over the decomposition of the scattered fields as the presence of a substrate makes it difficult to rigorously perform multipolar analysis using the scattered fields. When calculating the contributions associated with each multipole, the fields are considered to radiate into air, which makes the sum over multipole contributions (defined as total $Q_{\text {sca }}$ here) lower than the actual case. ${ }^{30}$

As can be seen in Fig. 2(a), the largest scattering efficiency occurs for $R=270 \mathrm{~nm}$, being determined mainly by contributions from the electric dipole (ED), magnetic dipole (MD), and electric quadrupole (EQ).

Next, the nonlinear polarization induced inside the nanoparticles is used as an input for the next electromagnetic simulations at the $\mathrm{TH}$ frequency to obtain the generated $\mathrm{TH}$ field. For centrosymmetric materials, such as silicon, THG is an essentially bulk phenomenon with negligible surface contribution. ${ }^{34}$ Here, we take into account only volume nonlinearities of silicon, and neglect its surface nonlinearities as of minor importance. Fig. 2(b) shows the rising trend of THG conversion efficiency at $R=270 \mathrm{~nm}$ with the decreasing gap size. Due to the precision limit of the EBL fabrication process, we use $G=20 \mathrm{~nm}$ to robustly create the nanodimer without linking the gap between the two disks by fabrication fluctuations. Fig. 2(c) shows the contributions from each multipole radiated at TH. The largest THG conversion efficiency also occurs at $R=270 \mathrm{~nm}$, corresponding to the typical resonant profile at a fundamental wavelength.

\section{Control of nonlinear radiation}

We measure the THG efficiency from our samples with increasing disk radii at a fixed gap size $G=20 \mathrm{~nm}$. We calibrate the cameras with a power meter. The measured THG conversion efficiency is shown with red solid dots in Fig. 2(d). The maximum efficiency reaches $2 \times 10^{-6}$. We also calculate the THG efficiency within the numerical apertures of the collecting objectives as utilized in our experiment. The respective theoretical dependence is plotted with a solid line. The position of the maximum THG conversion efficiency in Fig. 2(d) overlaps with the peak of the calculated linear scattering efficiency in Fig. 2(a).

Fig. 3( $(\mathrm{a}$ and $\mathrm{b}$ ) show the computed linear scattering spectra for the dimer with the optimized disk radius $R=270 \mathrm{~nm}$. We assume light normally incident from the air side with linear polarization that is either horizontal (a) or vertical (b). In Fig. 3 we see a pronounced peak in the scattering for horizontal polarization that is not present for vertical polarization. This modification of the scattering spectrum arises from coupling between the nanoparticles in the dimer. The spectral position of the peak matches the pump wavelength of our laser.
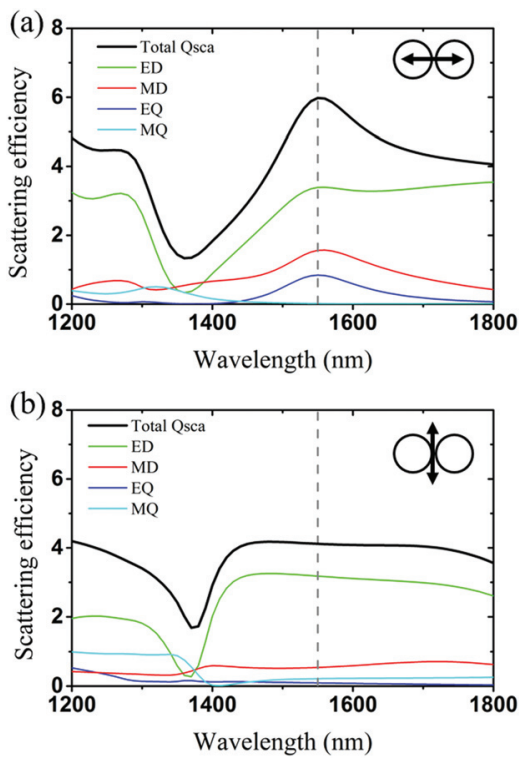

Fig. 3 (a) Calculated linear scattering efficiency and related multipolar decomposition for a silicon dimer with $R=270 \mathrm{~nm}$ and $G=20 \mathrm{~nm}$ excited by horizontally polarized (a) and vertically polarized (b) pump laser beams, respectively.
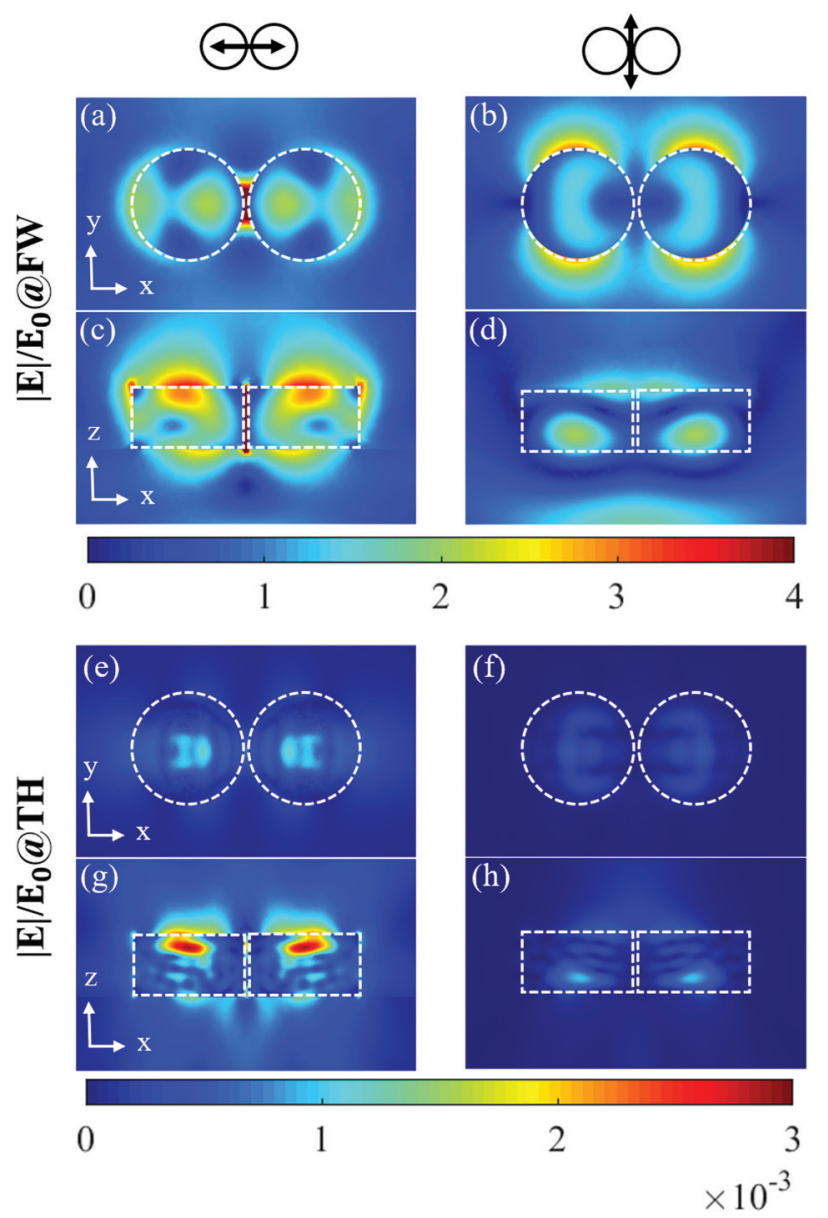

Fig. 4 Top and side views of the electric field distributions at fundamental wavelength (FW) and third harmonic (TH) in the silicon dimer antenna for two polarizations. The antenna has $270 \mathrm{~nm}$ disk radius and $20 \mathrm{~nm}$ interparticle gap. 
The far-field spectra are dominated by the following multipoles: ED, MD and EQ in Fig. 3(a) and ED, MD and magnetic quadrupole (MQ) in Fig. 3(b). All of them stem from the hybridization of electric and magnetic dipolar resonances of single scatterers, being qualitatively the same as described in ref. 26 and 28. Remarkably, the modes of coupled particles are known to play an important role in the analysis of the nonlinear response for both plasmonic ${ }^{35,36}$ and dielectric ${ }^{24}$ nanostructures.

The calculated electric near-field distributions at the fundamental and harmonic wavelengths for $x$ and $y$ polarized irradiance are depicted in Fig. 4. The FW near-field distributions are clearly seen to be determined by hybridized modes associated with electric and magnetic dipolar excitations inside the $\operatorname{discs}^{27,28,32}$ as shown in Fig. 4(a). For the $x$-polarized pump, the electric-field hotspot naturally occurs in the gap between two silicon nanodiscs. ${ }^{30}$ Strong field localization in coupled nanoantennas and electric field enhancement furthermore stimulate the nonlinear response in the nanoparticles. THG is predominantly governed by magnetic dipoles, ${ }^{23,24}$ as reflected in the symmetry of the generated TH field in Fig. 4. Because of the different physics compared to second harmonic generation (SHG) in coupled metal nanoparticles, ${ }^{35-37}$ no localization to (a)

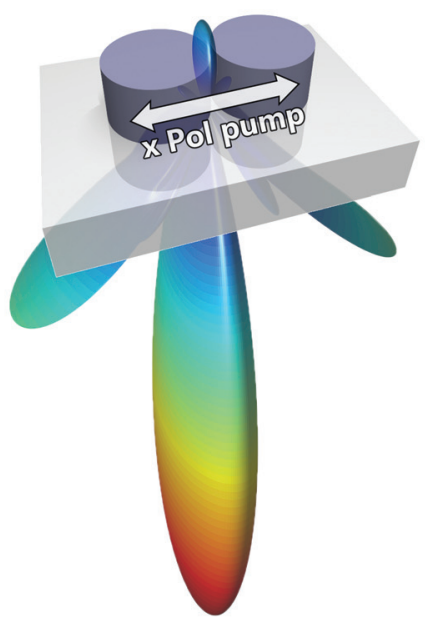

(c)

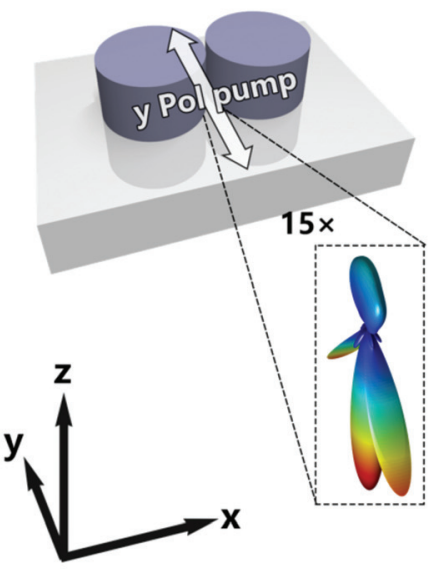

(b)

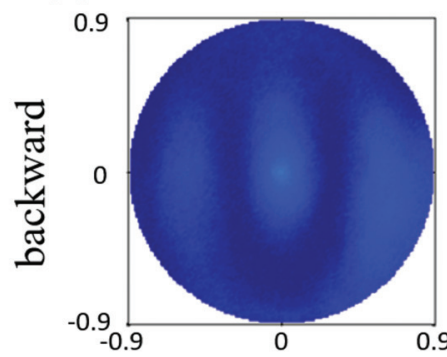

Experiment

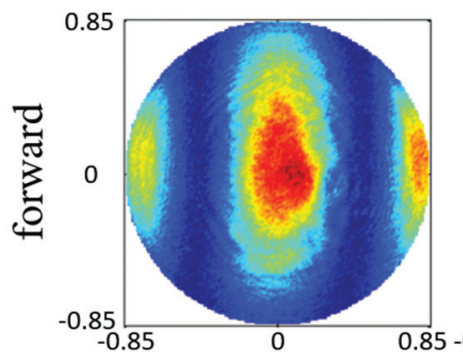

$-0.9$
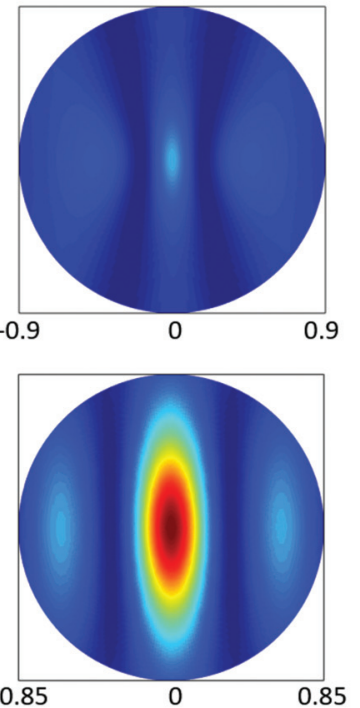

y-polarized pump

(d)
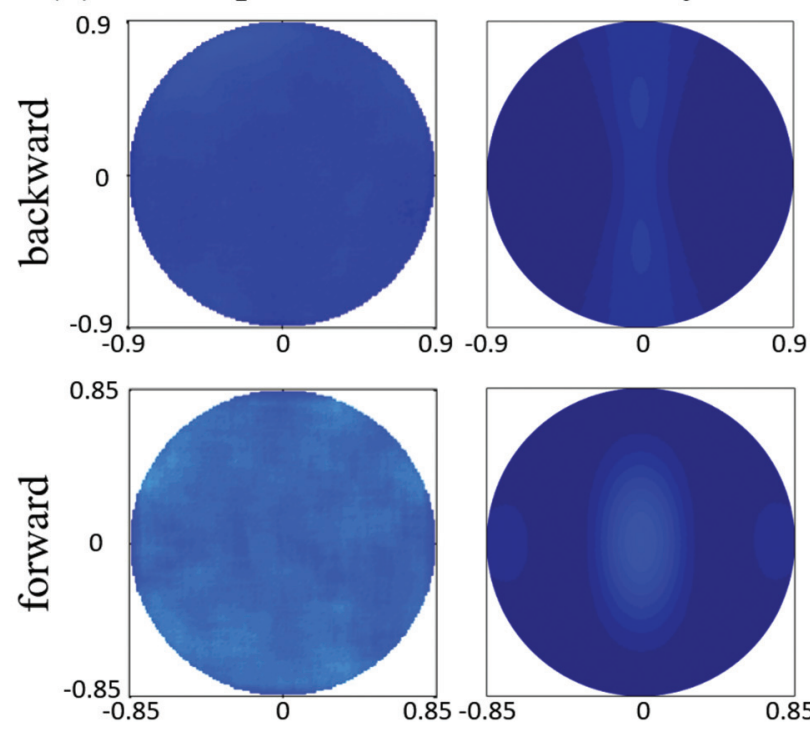

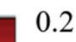

0.2

1.0

0.5

0.0

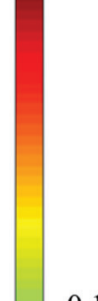

0.1

0.0

Fig. 5 Radiation patterns of THG for $x(a, b)$ and $y(c, d)$ polarized excitations. (a, c) Calculated three-dimensional directionality of TH emission. (b, d) Two-dimensional projections of three-dimensional directionalities measured and calculated within the numerical apertures of the objectives. The projections are shown in forward and backward directions with respect to the direction of the pump. 
the gap and far-field silencing ${ }^{35}$ of the nonlinear response is observed. As follows from Fig. 3 and 4, the linear scattering is quite sensitive to the polarization of the impinging light. The magnitude of the electric near-field and the induced magnetic dipole moments are stronger for the $x$-polarized pump promoting the largest coupling strength. Being raised to the third power in the nonlinear regime, this effectively switches THG on/off as the pump polarization is changed from $x$ to $y$.

To observe these phenomena in experiment, we measure the third-harmonic radiation patterns by capturing their directionality diagrams. This is done in forward and backward directions with respect to the direction of the pump. For this we project onto the cameras back-focal plane (Fourier space) images of our objective lenses. We perform matching numerical simulations where calculated $\mathrm{TH}$ far-field intensity distribution is projected onto the back focal plane. ${ }^{4,38}$ As shown in Fig. 5, the experimentally measured directionality of THG has the forward to backward ratio around $11: 1$, indicating highly directional nonlinear harmonic generation. The highly directional nonlinear scattering in Fig. 5(a) is defined by two higher-order electric multipoles EQ and EH. Numerical simulations predict a remarkable polarization-dependent contrast in the nonlinear signal: the third-harmonic power for the $x$-polarized pump is more than 30 times larger than the power for the $y$-polarized pump. The experimental measurements for the $y$-polarized pump appear below the noise level, but there is at least a $20: 1$ distinction ratio.

\section{Summary}

We have studied, both numerically and experimentally, the third-harmonic generation from all-dielectric dimer nanoantennas composed of two closely placed identical silicon nanodisks. We have observed strong nonlinear beam reshaping and optimized conversion efficiency (up to $2 \times 10^{-6}$ ), combined with highly directional harmonic generation characterized by the 11:1 forward-to-backward ratio. Furthermore, we have demonstrated the strong polarization dependence of the harmonic generation efficiency, characterized by more than $20: 1$ contrast of the THG intensity for the $x$ and $y$-polarizations, respectively. The capability of beam shaping in the nonlinear harmonic generation demonstrated here for the example of silicon nanodimers is expected to be a general feature of multipolar nonlinear effects, and it may pave a way toward a novel approach in the design of efficient and tunable unidirectional nonlinear light sources.

\section{Funding information}

This work has been supported by the Australian Research Council.

\section{Acknowledgements}

The authors acknowledge useful discussions with A. Fedyanin, M. Kauranen, and P. Urbach, and thank the ACT Node of the Australian National Fabrication Facility for a continuous support. We acknowledge the support from the Australian Research Council. D. A. S. acknowledges the support from the Russian Foundation for Basic Research (RFBR) (Grants 16-3200635, 16-02-00547). A part of this research was conducted at the Center for Nanophase Materials Sciences, which is a DOE Office of Science User Facility.

\section{References}

1 M. Kauranen and A. V. Zayats, Nonlinear plasmonics, Nat. Photonics, 2012, 6, 737-748.

2 J. Butet, P.-F. Brevet and O. J. F. Martin, Optical second harmonic generation in plasmonic nanostructures: From fundamental principles to advanced applications, $A C S$ Nano, 2015, 9, 10545-10562.

3 D. Smirnova and Y. S. Kivshar, Multipolar nonlinear nanophotonics, Optica, 2016, 3, 124.

4 L. Novotny and B. Hecht, Principles of Nano-Optics, Cambridge University Press, 2006.

5 N. I. Zheludev and Y. S. Kivshar, From metamaterials to metadevices, Nat. Mater., 2012, 11, 917-924.

6 M. Lippitz, M. A. van Dijk and M. Orrit, Third-harmonic generation from single gold nanoparticles, Nano Lett., 2005, 5, 799-802.

7 H. Aouani, M. Rahmani, M. Navarro-Cía and S. A. Maier, Third-harmonic-upconversion enhancement from a single semiconductor nanoparticle coupled to a plasmonic antenna, Nat. Nanotechnol., 2014, 9, 290-294.

8 B. Metzger, T. Schumacher, M. Hentschel, M. Lippitz and H. Giessen, Third harmonic mechanism in complex plasmonic Fano structures, ACS Photonics, 2014, 1, 471-476.

9 S. Kim, J. Jin, Y.-J. Kim, I.-Y. Park, Y. Kim and S.-W. Kim, High-harmonic generation by resonant plasmon field enhancement, Nature, 2008, 453, 757-760.

10 T. Hanke, J. Cesar, V. Knittel, A. Trügler, U. Hohenester, A. Leitenstorfer and R. Bratschitsch, Tailoring spatiotemporal light confinement in single plasmonic nanoantennas, Nano Lett., 2012, 12, 992-996.

11 M. Hentschel, T. Utikal, H. Giessen and M. Lippitz, Quantitative modeling of the third-harmonic emission spectrum of plasmonic nanoantennas, Nano Lett., 2012, 12, 3778-3782.

12 A. I. Kuznetsov, A. E. Miroshnichenko, M. L. Brongersma, Y. S. Kivshar and B. Luk'yanchuk, Optically resonant dielectric nanostructures, Science, 2016, 354, 2472.

13 A. I. Kuznetsov, A. E. Miroshnichenko, Y. H. Fu, J. Zhang and B. Luk'yanchuk, Magnetic light, Sci. Rep., 2012, 2, 492.

14 I. Staude, A. E. Miroshnichenko, M. Decker, N. T. Fofang, S. Liu, E. Gonzales, J. Dominguez, T. S. Luk, D. N. Neshev, I. Brener and Y. Kivshar, Tailoring directional scattering through magnetic and electric resonances in subwavelength silicon nanodisks, ACS Nano, 2013, 7, 7824-7832.

15 Y. H. Fu, A. I. Kuznetsov, A. E. Miroshnichenko, Y. F. Yu and B. Luk'yanchuk, Directional visible light scattering by silicon nanoparticles, Nat. Commun., 2013, 4, 1527. 
16 P. Albella, T. Shibanuma and S. A. Maier, Switchable directional scattering of electromagnetic radiation with subwavelength asymmetric silicon dimers, Sci. Rep., 2015, 5, 18322.

17 T. Shibanuma, P. Albella and S. A. Maier, Unidirectional light scattering with high efficiency at optical frequencies based on low-loss dielectric nanoantennas, Nanoscale, 2016, 8, 14184-14192.

18 M. R. Shcherbakov, D. N. Neshev, B. Hopkins, A. S. Shorokhov, I. Staude, E. V. Melik-Gaykazyan, M. Decker, A. A. Ezhov, A. E. Miroshnichenko, I. Brener, A. A. Fedyanin and Y. S. Kivshar, Enhanced third-harmonic generation in silicon nanoparticles driven by magnetic response, Nano Lett., 2014, 14, 64886492.

19 L. Carletti, A. Locatelli, D. N. Neshev and C. De Angelis, Shaping the radiation pattern of second-harmonic generation generation from AlGaAs dielectric nanoantennas, ACS Photonics, 2016, 3, 1500-1507.

20 V. F. Gili, L. Carletti, A. Locatelli, D. Rocco, M. Finazzi, L. Ghirardini, I. Favero, C. Gomez, A. Lemaître, M. Celebrano, C. De Angelis and G. Leo, Monolithic AlGaAs second-harmonic nanoantennas, Opt. Express, 2016, 24, 15965.

21 S. Liu, M. B. Sinclair, S. Saravi, G. A. Keeler, Y. Yang, J. Reno, G. M. Peake, F. Setzpfandt, I. Staude, T. Pertsch and I. Brener, Resonantly Enhanced Second-Harmonic Generation Using II-IấL̂SV Semiconductor All-Dielectric Metasurfaces, Nano Lett., 2016, 16, 5426-5432.

22 R. Camacho, M. Rahmani, S. Kruk, L. Wang, L. Xu, D. A. Smirnova, A. Solntsev, A. E. Miroshnichenko, H. H. Tan, F. Karouta, S. Naureen, K. Vora, L. Carletti, C. de Angelis, C. Jagadish, Y. S. Kivshar and D. N. Neshev, Nonlinear generation of vector beams from AlGaAs nanoantennas, Nano Lett., 2016, 16, 7191-7197.

23 D. A. Smirnova, A. B. Khanikaev, L. A. Smirnov and Y. S. Kivshar, Multipolar third-harmonic generation driven by optically induced magnetic resonances, ACS Photonics, 2016, 3, 1468-1476.

24 A. S. Shorokhov, E. V. Melik-Gaykazyan, D. A. Smirnova, B. Hopkins, K. E. Chong, D.-Y. Choi, M. R. Shcherbakov, A. E. Miroshnichenko, D. N. Neshev, A. A. Fedyanin and Y. S. Kivshar, Multifold enhancement of third-harmonic generation in dielectric nanoparticles driven by magnetic Fano resonances, Nano Lett., 2016, 16, 4857-4861.

25 G. Grinblat, Y. Li, M. P. Nielsen, R. F. Oulton and S. A. Maier, Enhanced third harmonic generation in single germanium nanodisks excited at the anapole mode, Nano Lett., 2016, 16, 4635-4640.
26 P. Albella, M. A. Poyli, M. K. Schmidt, S. A. Maier, F. Moreno, J. J. Sáenz and J. Aizpurua, Low-loss electric and magnetic field-enhanced spectroscopy with subwavelength silicon dimers, J. Phys. Chem. C, 2013, 117, 13573-13584.

27 J. Yan, P. Liu, Z. Lin, H. Wang, H. Chen, C. Wang and G. Yang, Directional fano resonance in a silicon nanosphere dimer, ACS Nano, 2015, 9, 2968-2980.

28 U. Zywietz, M. K. Schmidt, A. B. Evlyukhin, C. Reinhardt, J. Aizpurua and B. N. Chichkov, Electromagnetic resonances of silicon nanoparticle dimers in the visible, ACS Photonics, 2015, 2, 913-920.

29 S. Kruk, M. Weismann, A. Y. Bykov, E. A. Mamonov, I. A. Kolmychek, T. Murzina, N. C. Panoiu, D. N. Neshev and Y. S. Kivshar, Enhanced magnetic second-harmonic generation from resonant metasurfaces, ACS Photonics, 2015, 2, 1007-1012.

30 R. M. Bakker, D. Permyakov, Y. F. Yu, D. Markovich, R. Paniagua-Domínguez, L. Gonzaga, A. Samusev, Y. Kivshar, B. Luk'yanchuk and A. I. Kuznetsov, Magnetic and electric hotspots with silicon nanodimers, Nano Lett., 2015, 15, 2137-2142.

31 L. Wang, A. S. Shorokhov, P. N. Melentiev, S. Kruk, M. Decker, C. Helgert, F. Setzpfandt, A. A. Fedyanin, Y. S. Kivshar and D. N. Neshev, Multipolar third-harmonic generation in fishnet metamaterials, ACS Photonics, 2016, 3, 1494-1499.

32 J. van de Groep, T. Coenen, S. A. Mann and A. Polman, Direct imaging of hybridized eigenmodes in coupled silicon nanoparticles, Optica, 2016, 3, 93.

33 E. D. Palik, Handbook of Optical Constants of Solids, Academic Press, Boston, 1985.

34 J. E. Sipe, D. J. Moss and H. M. Van Driel, Phenomenological theory of optical second-and thirdharmonic generation from cubic centrosymmetric crystals, Phys. Rev. B: Condens. Matter, 1987, 35, 1129.

35 J. Berthelot, G. Bachelier, M. Song, P. Rai, G. C. des Francs, A. Dereux and A. Bouhelier, Silencing and enhancement of second-harmonic generation in optical gap antennas, Opt. Express, 2012, 20, 10498.

36 G. D. Bernasconi, J. Butet and O. J. F. Martin, Mode analysis of second-harmonic generation in plasmonic nanostructures, J. Opt. Soc. Am. B, 2016, 33, 768.

37 J. Butet, S. Dutta-Gupta and O. J. F. Martin, Surface secondharmonic generation from coupled spherical plasmonic nanoparticles: Eigenmode analysis and symmetry properties, Phys. Rev. B: Condens. Matter, 2014, 89, 245449.

38 M. A. Lieb, J. M. Zavislan and L. Novotny, Single-molecule orientations determined by direct emission pattern imaging, J. Opt. Soc. Am. B, 2004, 21, 1210. 